Preprint

UCRL-JC-134765

\title{
Automated Damage Onset Analysis Techniques Applied to KDP Damage and the Zeus Small Area Damage Test Facility
}

\author{
R. Sharp III, M. Runkel
}

This article was submitted to

$31^{\text {st }}$ Boulder Damage Symposium: Annual Symposium on Optical Materials for

High Power Lasers, Boulder, CO, October 4-7, 1999

U.S. Department of Energy

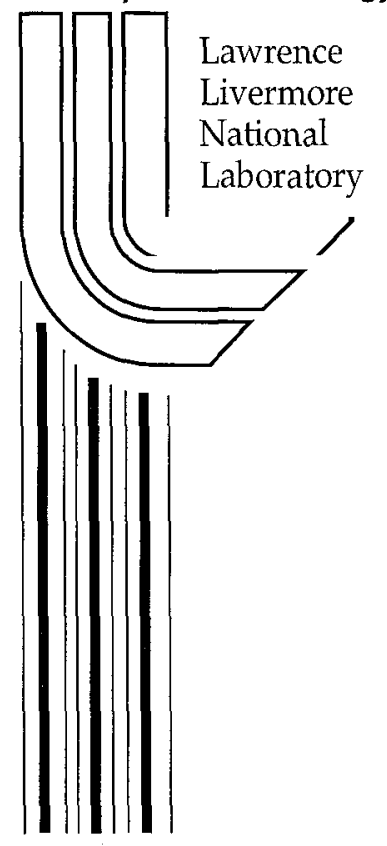

December 16, 1999 


\section{DISCLAIMER}

This document was prepared as an account of work sponsored by an agency of the United States Government. Neither the United States Government nor the University of California nor any of their employees, makes any warranty, express or implied, or assumes any legal liability or responsibility for the accuracy, completeness, or usefulness of any information, apparatus, product, or process disclosed, or represents that its use would not infringe privately owned rights. Reference herein to any specific commercial product, process, or service by trade name, trademark, manufacturer, or otherwise, does not necessarily constitute or imply its endorsement, recommendation, or favoring by the United States Government or the University of California. The views and opinions of authors expressed herein do not necessarily state or reflect those of the United States Government or the University of California, and shall not be used for advertising or product endorsement purposes.

This is a preprint of a paper intended for publication in a journal or proceedings. Since changes may be made before publication, this preprint is made available with the understanding that it will not be cited or reproduced without the permission of the author.

This report has been reproduced directly from the best available copy.

Available to DOE and DOE contractors from the Office of Scientific and Technical Information

P.O. Box 62, Oak Ridge, TN 37831

Prices available from (423) 576-8401

http://apollo.osti.gov/bridge/

Available to the public from the

National Technical Information Service

U.S. Department of Commerce

5285 Port Royal Rd., Springfield, VA 22161

http://www.ntis.gov/

OR

Lawrence Livermore National Laboratory

Technical Information Department's Digital Library

http://www.llnl.gov/tid/Library.html 


\title{
Automated damage onset analysis techniques applied to KDP damage and the Zeus small area damage test facility
}

\author{
Richard Sharp III and Mike Runkel \\ Lawrence Livermore National Laboratory \\ P.O. Box 808, L-250 \\ Livermore, CA 94550
}

\begin{abstract}
Automated damage testing of KDP using LLNL's Zeus automated damage test system ${ }^{1}$ has allowed the statistics of KDP bulk damage to be investigated. Samples are now characterized by the cumulative damage probability curve, or S-curve, that is generated from hundreds of individual test sites per sample. A HeNe laser/ PMT scatter diagnostic is used to determine the onset of damage at each test site. The nature of KDP bulk damage is such that each scatter signal may possess many different indicators of a damage event. Because of this, the determination of the initial onset for each scatter trace is not a straightforward affair and has required considerable manual analysis. The amount of testing required by crystal development for the National Ignition Facility (NIF) has made it impractical to continue analysis by hand. Because of this, we have developed and implemented algorithms for analyzing the scatter traces by computer.
\end{abstract}

We discuss the signal cleaning algorithms and damage determination criteria that have lead to the successful implementation of a LabView based analysis code. For the typical R/1 damage data set, the program can find the correct damage onset in more than $80 \%$ of the cases, with the remaining $20 \%$ being left to operator determination. The potential time savings for data analysis is on the order of $\sim 100 \mathrm{X}$ over manual analysis and is expected to result in the savings of at least 400 man-hours over the next 3 years of NIF quality assurance testing.

Keywords: KDP, bulk damage, automated onset, signal processing, damage detection

\section{INTRODUCTION}

Construction of the National Ignition Facility (NIF) at Lawrence Livermore National Laboratory (LLNL) has promoted intensive investigation into the manifestations of laser damage in the optical components of the laser. The DKDP triple harmonic generation crystal (THG, tripler) has been identified as a component that may be particularly susceptible to laser damage because it will be subjected to high average fluences. As a result, a significant effort to improve the damage performance of DKDP has been undertaken. This effort has yielded improved crystals through the use of continuous solution filtration, ultra-high purity starting salts and a number of other growth parameters. In order to give the crystal growers more complete feedback regarding the damage performance of their crystals, improved damage test technology was also required. The need for rapid turnaround with a statistically large number of test sites on the sample led to the development of the ZEUS automated damage test system ${ }^{1}$. This system consists of a commercially available, table-top Nd:YAG system operating primarily at $355 \mathrm{~nm}(3 \omega)$. Damage is detected by a photomultiplier tube that measures changes in the scatter signal from a $\mathrm{HeNe}$ laser that is co-linear to the $3 \omega$ damage beam. The system can test approximately 100 sites/hour. While we have automated damage detection and test execution, one area requiring direct operator involvement was the post-test analysis of the damage scatter traces, since damage onset and evolution can show substantial variability. Direct operator analysis of the individual damage traces is not a problem for small numbers of tests, however, prolonged analysis can result in a high level of tedium resulting in loss of objectivity and efficiency. Despite the signal variability in the damage trace data, determination of the individual damage onsets does lend itself to automation with the potential to save considerable amounts of time compared to that half hour required for manual analysis of the scatter traces. In the following sections we describe details of the ZEUS damage tester and the requirements for automated determination of the local threshold at a damage test site.

\subsection{The ZEUS automated damage test system}

The ZEUS damage test facility is a frequency tripled, 2 Joule Nd:YAG laser operating at $10 \mathrm{~Hz}$ with a $7.6 \mathrm{~ns}, 3 \omega$ pulse. It is capable of delivering $100 \mathrm{~J} / \mathrm{cm}^{2}$ of $3 \omega$ light in a 700 micron (FW@ $1 / \mathrm{e}^{2}$ ) spot to the sample. Operation of the system is via 
computer that controls the beam fluence at the sample by a variable attenuator, which consists of a rotating half-

waveplate/polarizer combination for energy control. The computer also controls sample translation (XY) stages and beamline shutters. A data acquisition card is used to record voltage readings from the damage detection diagnostic that consists of a 30 $\mathrm{mW} \mathrm{HeNe}$ probe laser and photomultiplier tube. This arrangement detects changes in the forward bulk scatter intensity from the crystal at approximately 45 degrees (see Figure 1.). The diagnostic allows the evolution of bulk scatter events at a test site to be recorded in real time as a function of the peak fluence in an individual laser pulse. The automation software allows user control over the number of sites tested, the sample grid (site spacing), the number of pulses per site and the fluence ramp rate $\left(\mathrm{J} / \mathrm{cm}^{2} / \mathrm{s}\right)$. In addition, voltage thresholds for damage detection and test termination as well as site acceptability are user controlled.

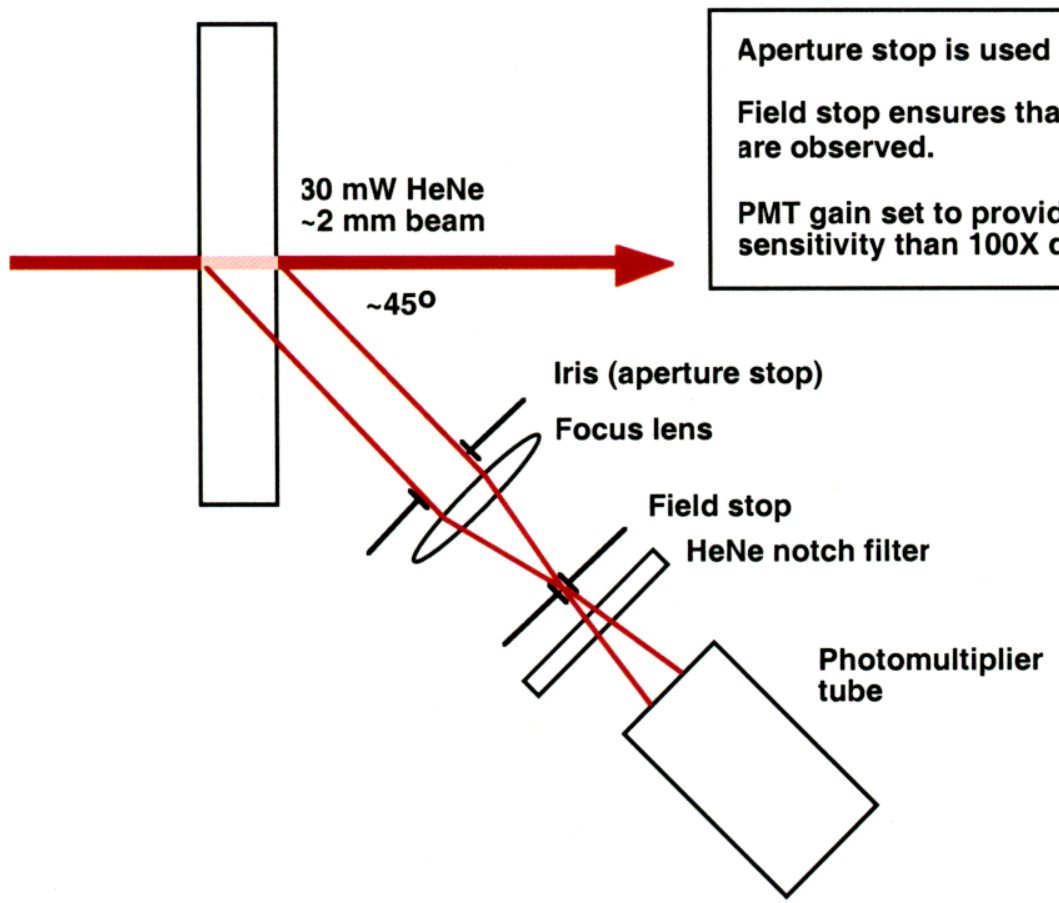

Figure 1. The Zeus bulk scatter diagnostic. The diagnostic detects changes in the bulk scatter of the test sample during irradiation. The aperture stop is used to control glare while the field stop is used to control the volume seen by the PMT. In this manner surface scatter can be virtually eliminated. The PMT gain is set empirically to provide comparable detection to $100 \mathrm{X}$ darkfield microscopy.

Our standard automated KDP damage test consists of exposing one hundred individual test sites to ramped fluence pulses until catastrophic damage occurs. For the 700 micron damage spot a grid spacing of $2 \mathrm{~mm}$ is used. Each test site is ramped from zero fluence up to damage at $0.5 \mathrm{~J} / \mathrm{cm}^{2} / \mathrm{s}$ increments. The beam diameter for the $30 \mathrm{~mW} H e N e$ probe laser is set to approximately $2 \mathrm{~mm}$. This spot diameter ensures approximately nearly uniform illumination of the damaged volume without interacting appreciably with previously tested sites. A scatter trace of typical events is shown in Figure 2. After sample irradiation the individual traces are analyzed to determine the earliest onset of damage at each site as shown in the figure. The associated onset fluences are recorded, sorted in ascending order and plotted. The sorted data can be interpreted as a failure probability curve. When the failure probability data is plotted versus the failure fluence the result is a characteristic " $\mathrm{S}$ " curve for the sample.

The automation, and manual post-test analysis software is written in LabView ${ }^{2}$. For the sake of convenience we chose to build the automated onset determination algorithms into the existing post-test analysis program. The choice of LabView as the programming language affected only the implementation of the algorithms 


\subsection{Characteristics of damage scatter traces}

Figure 2 shows the type of data generated by the Zeus automated damage test (ADT) system. Though somewhat idealized, this figure shows the main features of a typical scatter trace. There may be a slight reduction in the scatter voltage in the first few pulses due to either surface cleaning in the case of a dirty sample, or conditioning of micron scale inclusions in the case of a sample grown from unfiltered solution. The latter case has been virtually eliminated due to the use of continuous filtration of the solution during growth. Generally speaking, the scatter trace is relatively stable after the initial few pulses, however, there may be a slow oscillations in the signal due to DC power fluctuations in the HeNe laser or PMT power supply. In some cases a slowly increasing signal has also been observed. This has been attributed to an increase in the bulk scatter of the crystal that is not discrete in the manner of a micron-sized damage pinpoint resulting from the explosion of a small absorbing inclusion. The onset of bulk damage is detected as the abrupt increase in HeNe scatter from the "pinpoint" formed by the thermally initiated explosion of an absorbing nanoinclusion. It is a characteristic of KDP damage that once formed, these sites typically do not grow upon further exposure to $3 \omega$ light, and that further damage events are the result of microexplosions at other sites within the irradiated volume of the site. This is illustrated by the plateau in scatter voltage following each damage event. In addition to these features there can be considerable random noise on each scatter trace. The noise level can vary substantially depending on crystal surface finish quality and handling history in addition to the typical electrical line sources. Typical test traces are shown in Figure 3 to illustrate the variability of signal that can be found within the damage traces for a single test.

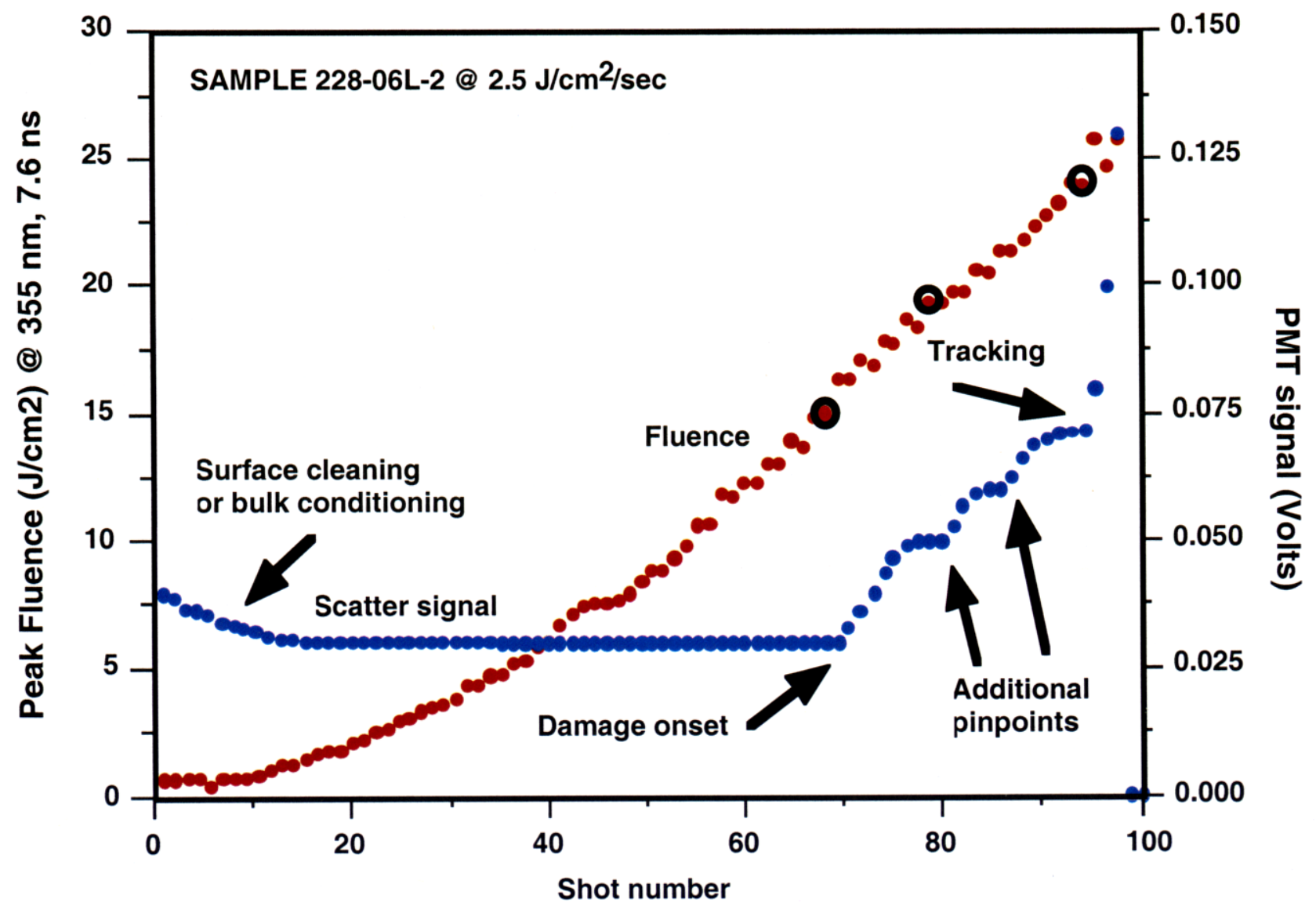

Figure 2. Typical scatter response from the bulk scatter diagnostic as a function of incident fluence. Features include surface cleaning/bulk conditioning, stabilization of the scatter signal until the formation of the first pinpoint, the formation of additional pinpoints up to catastrophic damage (tracking). 

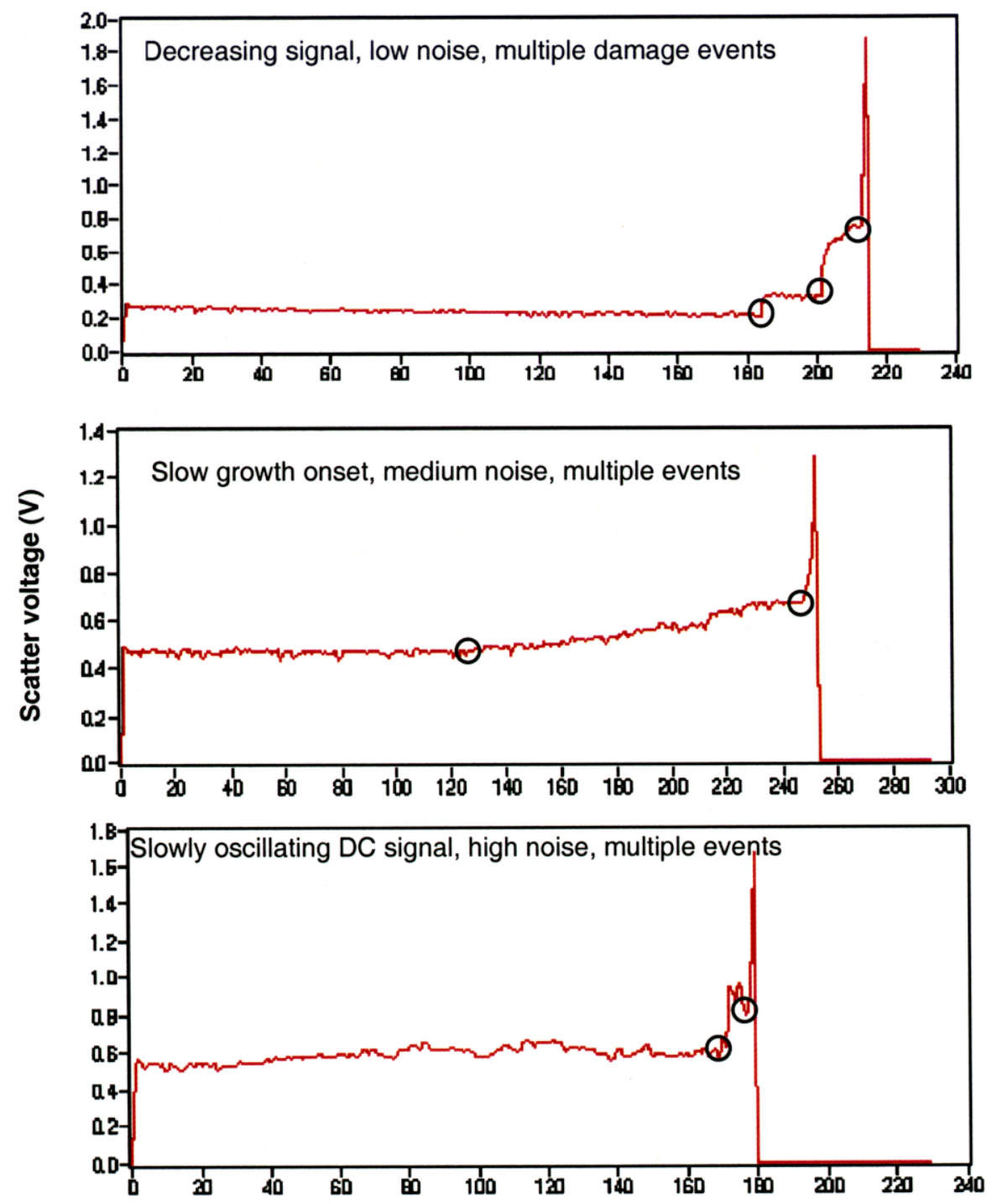

Shot number

Figure 3. Three scatter traces from the same KDP R/1 automated damage test. The traces show the variability in sample response that must be accounted for by damage onset determination algorithms. Circles indicate damage events.

\section{AUTOMATED ONSET DETERMINATION ALGORITHM}

Our approach to automated damage onset determination involves the following steps that will be discussed in detail below. First, the noisy signal is smoothed using a method we call "linear reconstruction". This method is based on recursive filtration ${ }^{3}$ of the input signal to achieve the desired reduction in sample noise while maintaining signal integrity. A similar program has been previously reported by Crochiere and Rabiner ${ }^{4}$, but was unknown to us during our program development.

After the smoothed scatter trace is obtained, it is compared to the actual signal to establish an average voltage baseline and noise level. The smoothed signal is next analyzed for damage onset events. Our criterion for damage onset events is a rise in smoothed signal above the baseline noise level. Since there can be multiple damage events in a trace, we search backwards from the highest signal level to determine the lowest onset event. The chosen onset event is evaluated by checking the local 
variance about the point. This process is repeated for each scatter trace in the data set and the onset data is used to build the damage S-curve.

\subsection{Signal cleaning by linear reconstruction}

The first task in determining the local damage onset for a scatter trace is to smooth the signal. This is done by a combination of cumulative, running signal averaging (with boundaries) and signal decimation and linear interpolation. The running signal averaging is done according to the prescription $\mathrm{V}_{\text {ave }}=\left(\Sigma \mathrm{V}_{\mathrm{n}}\right) / \mathrm{N}$ and $\mathrm{V}_{\mathrm{b}}=\mathrm{V}_{\text {ave }} 10^{\mathrm{kN}}$ where $\mathrm{V}_{\text {ave }}$ and $\mathrm{V}_{\mathrm{b}}$ denote the average and boundary signal voltages, $\mathrm{k}$ is a constant that was empirically determined during program development, and $\mathrm{n}$ is the shot number. $\mathrm{N}$ represents the total number of shots in a trace currently under consideration. As data smoothing by running average progresses, the software checks for points in the data where $V_{n}$ exceeds $V_{n} \pm 0.5 * V_{b}$. If the data lies within these boundaries, $\mathrm{N}$ is incremented by one, else $\mathrm{n}$ is reset to 2 and the running average starts over. The boundaries are a preliminary estimate of the noise, and have the property of expanding as $\mathrm{N}$ increases (locking in a steady signal). Determining the noise level further requires the smoothed signal. This averaging process is highly sensitive where the signal changes quickly and less so in static regions, however, it has the effect of leaving the averaged signal with steps. To eliminate this problem, the original signal is decimated where every $i^{\text {th }}$ element is picked and the signal is reconstructed to its original length based on linear interpolation of the "skeletonized" signal. The software compares the averaged and reconstructed signals to the original on a point-by-point basis and keeps the point closest (i.e. with the minimum deviation) to the original data. It is possible to obtain points from both the running average curve and the linearly reconstructed curve. This combined signal is then used as input and the entire running average /linear reconstruction comparison is repeated. The resultant signal from the second pass is then compared to the original data and the processed signal closest to the original data is kept. We have empirically determined that three passes of the averaging and linear reconstruction process is sufficient to remove the high frequency noise component from the data. An example of this process is shown in Figure 4.

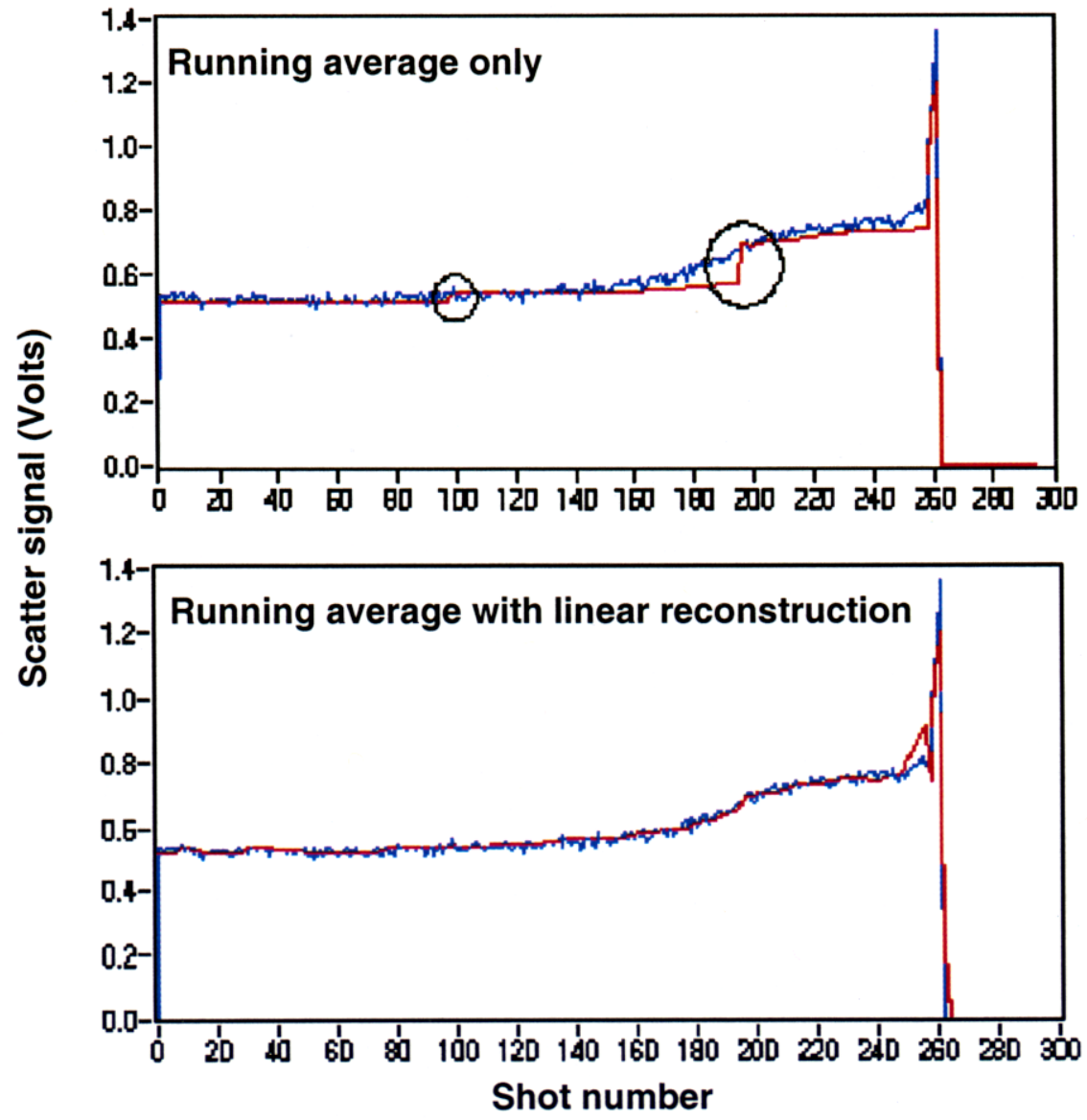

Figure 4. Results of data smoothing by running average and linear reconstruction. The top plot shows the results of running average only on a typical scatter trace, while the bottom plot shows the results of combining the two methods. 


\subsection{Determination of the baseline noise level and search for onset events.}

The baseline noise level is required to determine when the signal rises above the noise. We obtain the baseline noise signal using the formula $\mathrm{V}_{\text {noise }}=\Sigma\left[(\text { Signal-Average })_{n}{ }^{2}\right]^{0.5} / \mathrm{N}$. However, to keep the baseline noise value representative of the higher frequency noise component, we only calculate it from the first $15 \%$ to $65 \%$ of the curve. This range was chosen because it typically lies in the flat portion of the data between the regions where surface cleaning and damage occur for the majority of scatter traces encountered. Note that this is not a cumulative running average as in the previous section. Once the baseline noise level is determined the search for damage onset events can be done. This consists of determining the maximum voltage level in a scatter trace and searching backwards from it to find the point where the smoothed signal crossed the baseline noise level. Once a preliminary onset point is found, a number of acceptance criteria are applied to the data locally. Of particular importance is an increase in the local slope surrounding the onset point. Regions prior to and surrounding the preliminary onset point are examined for a transition from a flat to a sloped region and are rejected if there is no change. Also, estimates that occur many shots before the maximum voltage are rejected as these events are usually difficult to classify. The results of applying the noise baseline and backward search are shown in Figure 5.

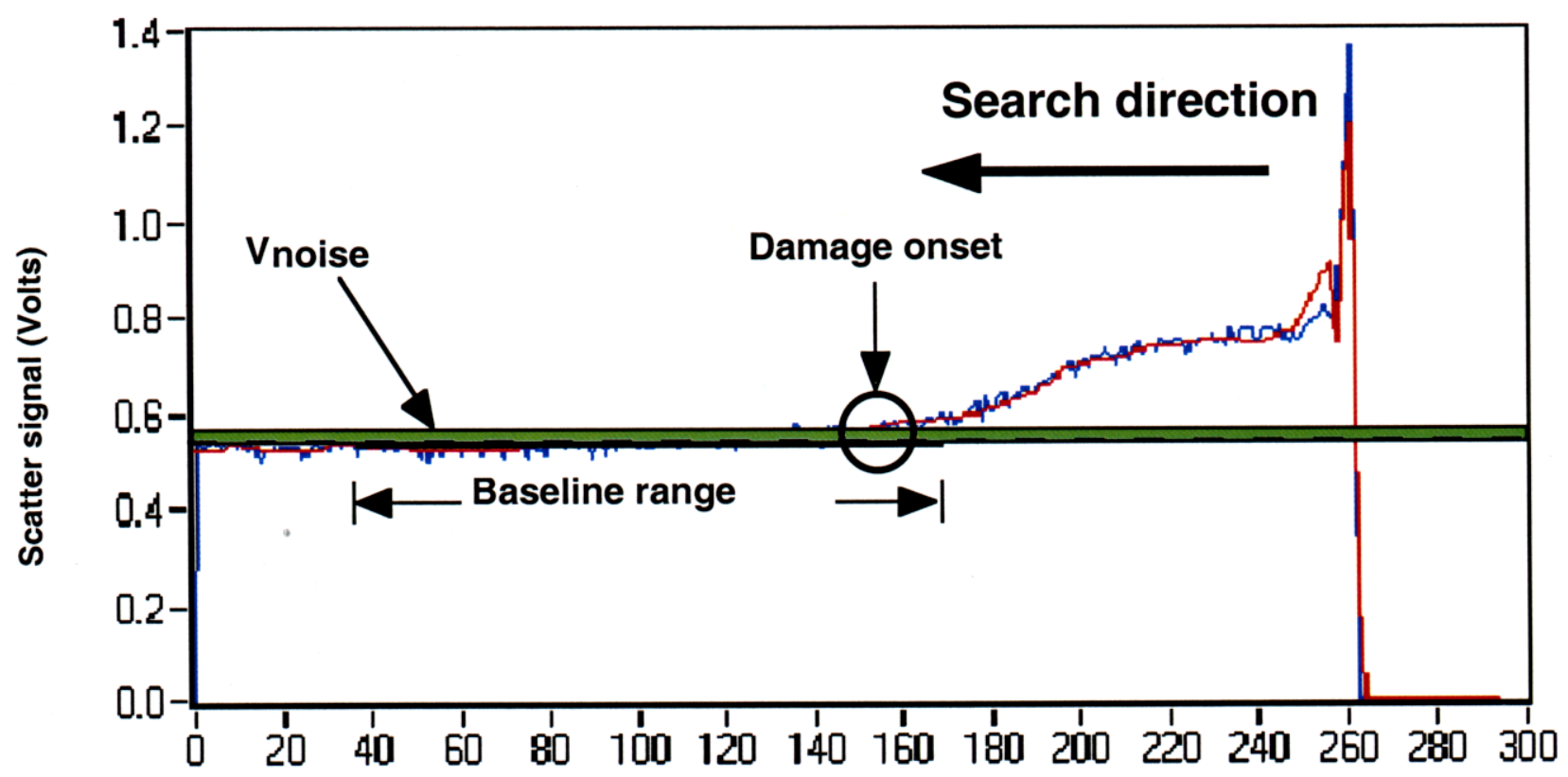

Shot number

Figure 5. A typical scatter trace showing the application of data smoothing, baseline noise determination and backward search to determine the damage onset. Once the shot number for onset is determined, the fluence corresponding to that shot is identified as the damage threshold for the test site. Analysis of the complete set of scatter traces and accompanying fluence data allows the failure probability curve to be constructed.

Application of this procedure to all test sites allows the cumulative failure probability (S-curve) to be constructed. The accuracy of the S-curve generated by the algorithm is determined by how well it compares to curves generated by hand analysis of the data. So, prior to entrusting the automation software with unchecked generation of S-curves, we performed numerous point-by-point comparisons of the automated and hand analyzed data. An example is shown below in Figure 6. 
DKDP576
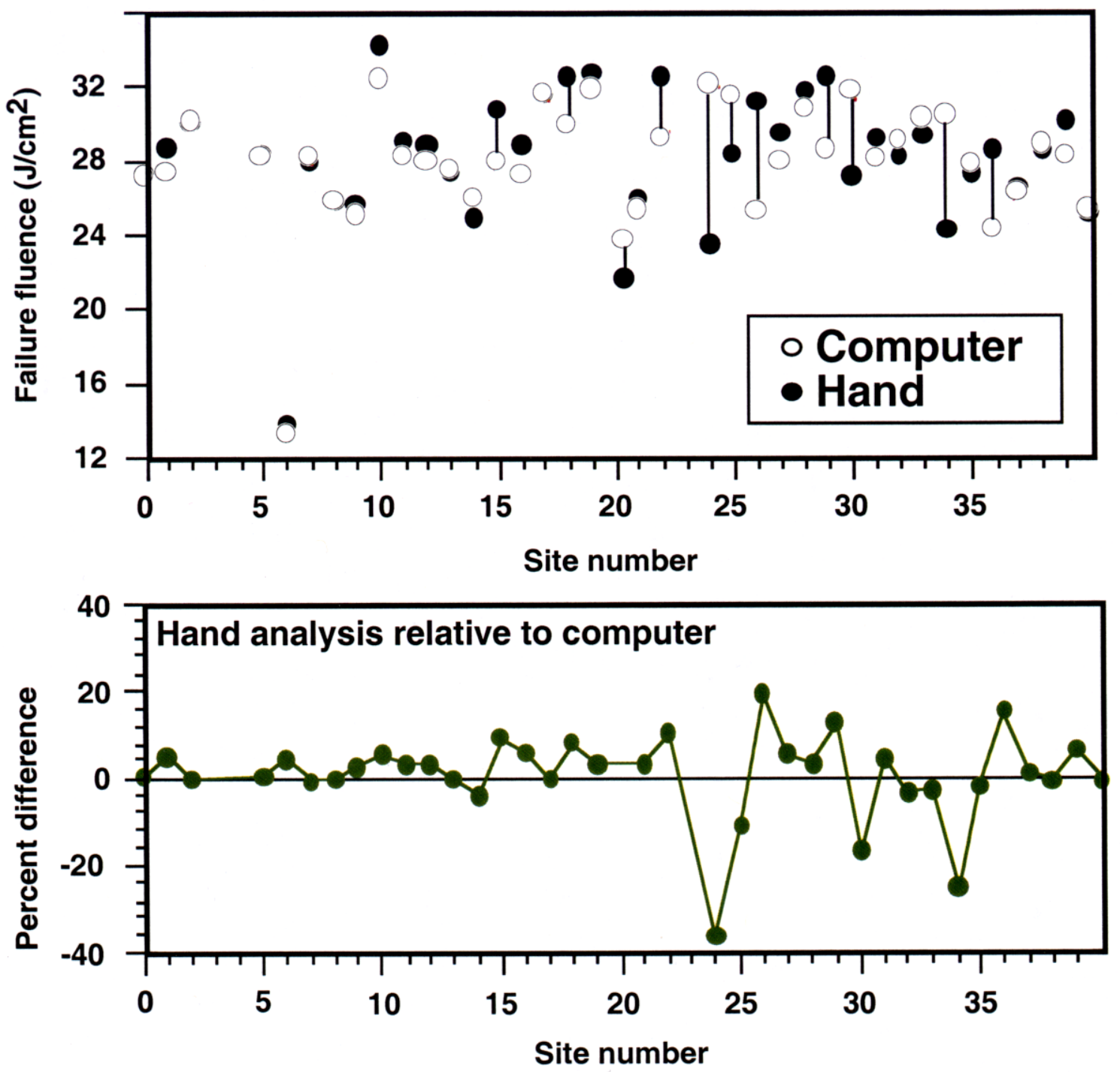

Figure 6. Comparison of computer and hand generated damage onsets in a site-by-site manner for damage onset data from rapid growth sample DKDP576. The top figure shows a direct comparison of the failure fluence picked by the computer and that picked by hand. The data pairs that show significant differences are connected by a line. The bottom figure shows the percent difference in computer vs. hand analysis for each point in the data set. In general, the difference in onset determination is less than 10 percent, however there can be deviations as large as $40 \%$.

We find that in general the computer chooses the damage onset at a slightly lower fluence than determined by hand analysis, however this is not always the case. Typically, the difference between computer and hand analysis is less than 10 percent, which is within the fluence measurement precision of $\pm 15 \%$ for our system. Thus, we do not expect smaller percent differences to greatly impact the S-curve generated from the data. As shown in Figure 7, the overall shape of the failure distribution does not change substantially due to the slightly more conservative onset determination by the computer and the occasional large difference between computer and hand analysis. Damage events at low fluence appear in the tail of the Scurves and have a greater effect on the shape of the curve than those that appear in the body of the curve. These low fluence events often have high noise levels or other features that confuse the algorithm and are therefore passed along to the user for hand analysis. The undetermined events are significant and need to be included in the S-curve, making the user's role indispensable. 


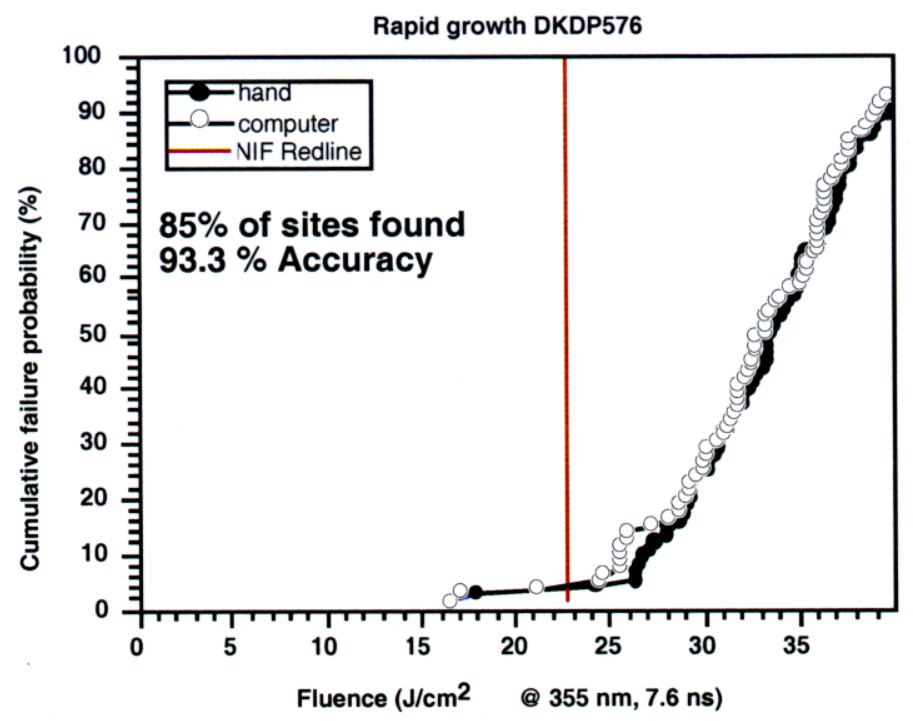

Figure 7. Damage probability curves constructed from computer and hand analysis of scatter traces for rapidly grown sample DKDP576. The computer generated curve shows a slightly lower onset fluence on a point-by-point basis. For this sample the computer determined onsets for $85 \%$ of the sites and did so with $93.3 \%$ average accuracy.

Comparison of computer and hand analysis on over 20 samples indicates that the average success rate of the computer in determining a damage onset is $85 \%$, meaning that the computer rejects $15 \%$ of the test sites which then require determination of the onset by the operator. Of the $85 \%$ of sites for which a threshold is found, the average percent deviation from the hand analysis is approximately $8 \%$ with corresponding accuracy of $92 \%$. Software parameters have been set to yield a slightly lower onset, however; overall this is only a "quasi-systematic" effect because in some instances computer determined thresholds can differ significantly from hand analysis. As long as these large deviations are infrequent the cumulative failure distribution is not significantly altered. Given the overall accuracy of the method, we can expect that approximately one curve out of twelve will deviate significantly from what would have been determined through hand analysis. This number can be reduced by comparing the automated data to on-line observations that are recorded for each test.

Implementation of the onset determination algorithms describe here has resulted in a significant reduction in data analysis time. For a 100 site damage test, hand analysis can take up to one hour. This time has been reduced to approximately 5 minutes with the automated onset determination software. This significantly increases the amount of data that can be collected and efficiently analyzed, and as importantly reduces operator fatigue and tedium which comes with intensive data analysis.

Work performed under the auspices of the United States Department of Energy by Lawrence Livermore National Laboratory under Contract No. W-7405-ENG-48.

\section{REFERENCES}

1) M. Runkel, M. Yan, J. DeYoreo, N. Zaitseva, "The effect of impurities and stress on the damage distributions of rapidly grown KDP crystals," Symposium on Laser Induced Damage in Optical Materials, SPIE Proceedings, Volume 3244, 1997

2) LabView version 4.1, National Instruments, Austin TX, 78759, 1998

3) K. G. Beauchamp, Signal Processing - Using Analog and Digital Techniques, John Wiley \& Sons, New York, 1973

4) R. E. Crochiere, L. R. Rabiner, "A program for multistage decimation, interpolation and narrow band filtering," Programs forDdigitalSsignal Processing, C. J. Weinstein, editor, IEEE Press, New York, 1979 